\title{
Frozen hydrated bloc-face investigation of tissue for Cryo SEM
}

\author{
T. Richter*, M. Sattler*, R. Wiesendanger**, K.-P. Wittern*, and R. Wepf*; *Beiersdorf AG, \\ R\&D cosmed, Analytical Microscopy Dept., Unnastr. 48, 20245 Hamburg, ${ }^{* *}$ Institute of \\ Applied Physics, University of Hamburg, Jungiusstr. 11, 20355 Hamburg, Germany
}

Scanning electron microscopy (SEM) is a versatile technique for analysing biological complex structures such as human skin. However, one of the most significant problems in imaging biological specimens in EM is their inherent water content, which is incompatible with high vacuum. Different approaches have been established to overcome this problem: either removal of the water by chemical fixation and dehydration, its physical immobilization by freezing or environmental SEM. In our study, the thickness of the outermost cell layers (the "Stratum Corneum", SC) of human skin had to be investigated at various hydration conditions. Since the thickness of these cells depend on their hydration state ${ }^{1}$ and the different layers of the SC are not accessible by an ESEM approach, a protocol had to be established, which preserves the hyrated state of the tissue for high resolution imaging.

Routinely samples are rapidly frozen by "plunge freezing", i.e. by plunging $3 \mathrm{~mm}$ skin biopsies into liquid ethane. This fast and reliable procedure provides a high throughput at sufficient freezing quality (for specimens with less than $20 \%$ water content such as the SC). Compared to high-pressure-frozen control samples, minor freezing artefacts are found, however, thickness measurements are not biased by these artefacts. In order to prevent alterations of cell volume or shape, we do not substitute or extract the immobilised water but established a cryo preparation chain preserving the frozen hydrated state of the specimen until imaging ${ }^{2}$.

Freeze-fracturing of these samples resulted in very rough surfaces, preventing reliable cell size measurements. To avoid fracture planes, we use an ultramicrotome with a trimdiamond knife to crossect the samples, revealing absolutely flat blocface planes (Fig.1). The whole cutting procedure takes place at $-130^{\circ} \mathrm{C}$ in nitrogen atmosphere, preventing recrystallisation and contamination with water from the air. Using a feed of $50 \mathrm{~nm}$, we cut several $10 \mu \mathrm{m}$ into the skin. This fine knife-movement in combination with a low $(0.4 \mathrm{~mm} / \mathrm{s})$ cutting-speed results in a very even and homogeneous sample surface (Fig. 1).

In order to achieve appropriate resolution for cell size quantification, a scanning electron microscope (LEO Gemini 1530), equipped with a cold stage at $-125^{\circ} \mathrm{C}$, is used to investigate the samples. To enhance the contrast of fine corrugations, we apply a low angle unidirectional platinum-carbon shadowing at an elevation angle of $30^{\circ}$, followed by a carbon backing to reduce electric charge ${ }^{3}$.

The different cell layers of the skin can be clearly resolved (Fig. 2), as well as further structures, e.g. hairs and sweat glands. Having established a pure physical fixation and preparation protocol for human skin, we are now able to investigate skin layers at various hydration conditions after a defined swelling procedure. Hence measurements of corneocytes thickness and reorganisation of cell content becomes possible. 


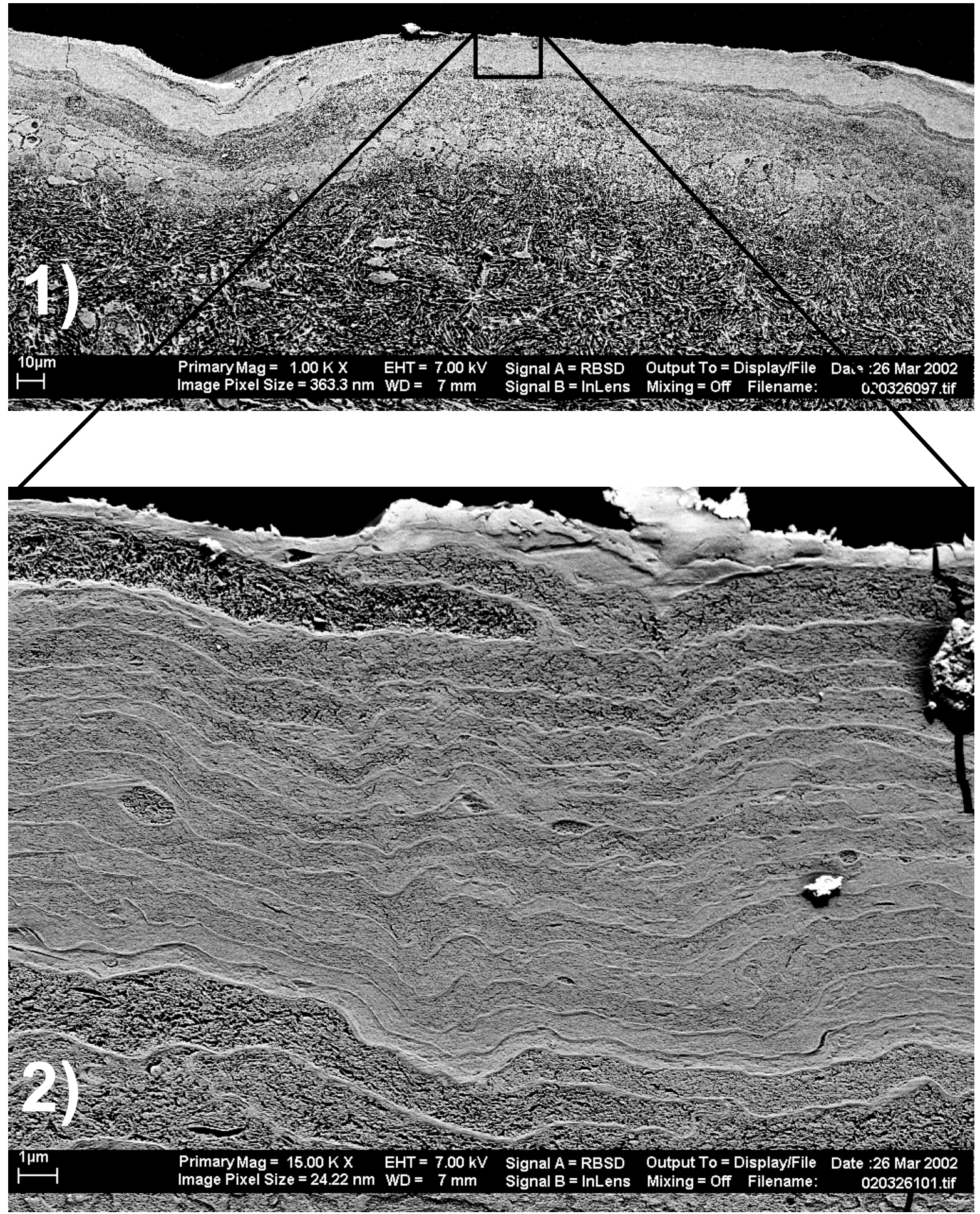

Figure 1: an example of a bloc-face of human skin

Figure 2: at higher resolution, the cell boundaries in the SC can be easily resolved References

${ }^{1}$ T. Richter et al., Applied Physics A 72, 125-128 (2001)

${ }^{2}$ M. Ritter et al., Microscopy \& Microanalysis 5 (Suppl. 2: Proceedings), 424-425, (1999)

${ }^{3}$ P. Walther et al., Scanning Microscopy, Vol. 5, No. 2, 301-310 (1991

This work was supported by a German Government BMBF grant 13N7695/9 